Research Article

\title{
Realization of Mobile Education Resource Sharing Method Based on Wireless Broadband Connection
}

\author{
Yongli Wu ID $^{1,2}$ and Jingliang Chen ${ }^{1}$ \\ ${ }^{1}$ Business School, University of Shanghai for Science and Technology, Shanghai 200093, China \\ ${ }^{2}$ East China Normal University, School of Communication, Shanghai 200093, China \\ Correspondence should be addressed to Yongli Wu; wuyongli@shmy.edu.cn
}

Received 4 August 2021; Revised 24 August 2021; Accepted 28 August 2021; Published 7 September 2021

Academic Editor: Mian Ahmad Jan

Copyright (c) 2021 Yongli Wu and Jingliang Chen. This is an open access article distributed under the Creative Commons Attribution License, which permits unrestricted use, distribution, and reproduction in any medium, provided the original work is properly cited.

\begin{abstract}
Educational resource sharing among students is always an essential component for the development of education level. With the progress of digital and communication networks among institutions, the use and sharing of high-grade educational resources can reach a new level. This paper proposes a new platform for mobile educational resource prediction and sharing via wireless broadband access technology. The architecture of the resource sharing system comprises resource, management, and service layers. An evaluation index system is established to assess the quality of the resource, and a detailed description of the resource assessment methods is presented. Moreover, the social impacts on the allocation of resources and similarities between the social metrics such as response time, success rate, and resource acquisition were investigated. The system has the potential to provide a high success rate and low response time. The system proposed in the paper will stimulate teamwork between teaching groups and increase the ability of resource sharing and optimization more comprehensively.
\end{abstract}

\section{Introduction}

Recently, the demand for high-grade educational resources has been rapidly increasing worldwide, and the application of information technology has enabled the elevation of education and encouraged education revolution in numerous fields. However, the present methods of educational resource sharing are still at an initial stage. The most widely used method of educational resource sharing nowadays is an online education platform based on cloud computing, such as Coursera, Udemy, FutureLearn, Pluralsight, and Moodle [1]. These online platforms virtualize educational resources and systems and deploy them on the cloud platform which enables users all over the world to get access to such resources. Compared with conventional classroom learning, network distance learning provides incredible advantages with the richness of educational resources, sharing, interactivity, and teamwork of teaching activities [2]. Through these learning communities, resources can be shared, interactive communication can be carried out, and cooperative learning relationships can be established. Many communities show strong interaction, and the online Q\&A of teachers and online communication of learners play a great role in promoting learning [3].

Mobile Notes is one example where technology is used in educational institutes to improve students' learning experience [4]. In this type of project, digital whiteboard and Personal Digital Assistant (PDA) devices are used by the pupils to provide data exchange and interaction. Educational information is shared in an asynchronous mode among the students through the use of a central database. Although a centralized asynchronous solution is beneficial for sharing educational information in several situations, these methods have a disadvantage that information is not shared instantly. Another educational resource project named Collpad [5] provides mobile-to-mobile communication for learning in an indoor environment. The system uses PDAs for communications for teachers and pupils and is based on WiFi connections in indoor environments. An educational resource sharing system based on combining several devices interfaces is presented in [6]. This system enables a user to combine several device interfaces and to connect seamlessly 
with one multidisplay device. The system employed a layered architecture and is confined to LAN as the communication channel. Didac et al. [7] proposed an education resource learning system comprised of aggregated mobile devices, forming organizations. These aggregated devices provide a new mechanism that facilitates the design of mobile learning activities offering a virtual complex device that combines the features of several mobile devices. Hou et al. [8] developed a method for educational resource sharing based on blockchain. The system was able to connect learners all over the world and provide access to high-quality educational resources. Moreover, the system has the potential to eliminate the trust barriers between higher educational institutions and social institutions and can well protect personal privacy. Yao and Xiong [9] proposed a cloud-based educational resource sharing model based on the analysis of related application status. The model comprises resources, service, and management layers. An assessment index was also constructed to evaluate the quality of the resource and the detailed description of the resource assessment methods. The model was effective to promote sharing of information resources and educational applications. Yalcin et al. [10] proposed a new model using virtualization technology for promoting educational materials using the computer network. The network topology consisted of real hardware instead of virtual ones, which distinguished it from similar topologies. This model can guide educational network and system managers to develop and use a real educational network and system management platform.

Conventional educational resource sharing methods use storage mode systems based on contents [11]. Data and information resources are stored on the storage server. If the server provides data for multiple users, a large-capacity hard drive will be required to store all types of educational resource files [12]. For colleges and universities, the recent growth of high-speed digital networks offers not only simple access to more efficient computing but also a new capability and an opportunity to access educational resources [13]. In this paper, a new algorithm is developed for mobile learning community resource sharing using broadband wireless connections. The system can provide high success rate and low response time while sharing educational resources among users. We also analyzed the impact of unequal distribution of resources on society and examined the similarity between response time and social indicators such as resource access.

The remaining sections of the paper are ordered as follows. Section 2 provides background and explains the architecture of the proposed education resource sharing system. The resource sharing algorithm is discussed in Section 3. In Section 4, the results are presented, and finally the conclusion is given in Section 5.

\section{Theoretical Analysis}

2.1. Social Network Analysis. Teacher communities are believed to contribute to improvements in the practices of teaching and research as well as to the collective capacity of educational institutions. The majority of teacher communities share educational resources in real-world environments and via online networks. Previous research shows that online networks may be an effective technique to promote collaboration and sharing of educational contents [14]. This study collected collaboration data from community members obtained through long-term experimental observation and recording. By examining the response time of members to acquire resources in the mobile learning community, recommendations that are more conducive to resource sharing can be made to promote the better development of the mobile learning community.

In the virtual learning community, the user relationship graph is formed by the friends between users [15]. After community division, there can be several subcommunities. The similarity between two users is calculated in the subcommunity, and the learning resources for the user's friends are calculated. Although learners in the virtual learning community form a social circle, there may be close and distant relationships among people in the social circle. People who have different number of friends in common have different chances of becoming friends, even if they are in the same social circle. Therefore, the affinity between learners can be obtained through similarity, thus generating a recommendation. In a mobile learning community, the learner can be connected on the cable to connect in the virtual community as well as through mobile devices using wireless or Bluetooth technology. For example, the function of WeChat is to look for someone near, so there is a high probability to become friends and share educational resources.

2.2. Network Centrality Analysis. Network centrality is a basic concept used to distinguish the status of community members in the network [16]. The higher the degree of centrality among individuals, the closer the relationship among the members of the community. If a member is directly related to a majority of the other members, then that member is a very important node in the process of community information transmission, playing a key role in interpersonal relationships, resource possession, and knowledge dissemination and sharing. On the contrary, if a member is in a marginal position in the network, he may acquire a lot of information, but he cannot play an important role in the transmission of information, and his position is not conducive to his contribution to the construction of group knowledge. The central potential is a quantification of the position of the local group in the community and represents the overall integration or consistency of the graph [17].

2.3. Network Density Analysis. Network density indicates the degree of interaction among the members of a community. A high density indicates more connections among members of a community, while a low density means fewer connections among members. The possible value of network density is between 0 and 1 . If there is a direct relationship between each pair of members in the network community, 
the maximum value of network density is 1 , but in most cases, the value of network density is less than 1 [18].

The network density is the ratio of the actual number of connections to the number of possible connections. Suppose that the interaction among the members of a community is represented by a directed graph " $G$ "; then, the density " $D$ " can be calculated as

$$
D=\frac{n}{N(N-1)},
$$

where $n$ is the number of actual connections and $N$ is the number of actors. Density $D$ shows the degree to which nodes are interconnected and can be used to measure the speed of information exchange.

2.4. Architecture of the Proposed Model. The overall architecture of the proposed intelligent distance education resource sharing platform is shown in Figure 1. The model comprises resource layer, management layer, and service layer. These layers are described in the following section [19].

(1) Service layer: this layer is mainly responsible for providing direct access to resource sharing services and is responsible for data storage. It also provides high storage capacity. This layer provides access to the shared resources using mobile phones, pads, computers, and other devices.

(2) Management layer: this layer is responsible for providing the system management functions, adopts cloud platform architecture, and simplifies the management process (by providing hardware equipment, storage, and operation management) and education managers to manage platform resources (including service, accounts, and resources). After applying for and obtaining a unified ID, each branch can access the resource pool and obtain corresponding services.

(3) Resource layer: in this layer, teaching resources and learning resources are summarized through unified interfaces. Sharing of teaching resources is realized through resource integration, and the unified storage of integrated teaching resources is used to effectively solve the problem of teaching resource management.

2.5. Information Storage. Distance education resource sharing platform based on cloud technology belongs to a large data center platform rather than a single commercial web server [18]. It consists of data center resources distributed in multiple regions and borrows a high-speed communication network as a platform. It combines all the control nodes of a university to the regional cloud data center to transmit teaching resources and realize the automatic transmission function of data among different cloud data centers. The central platform has the privileges to assign levels to users in provinces and cities. Combined with the addresses of accessing users, the data center is locked to further improve the data transmission efficiency. By modeling the resource sharing platform with

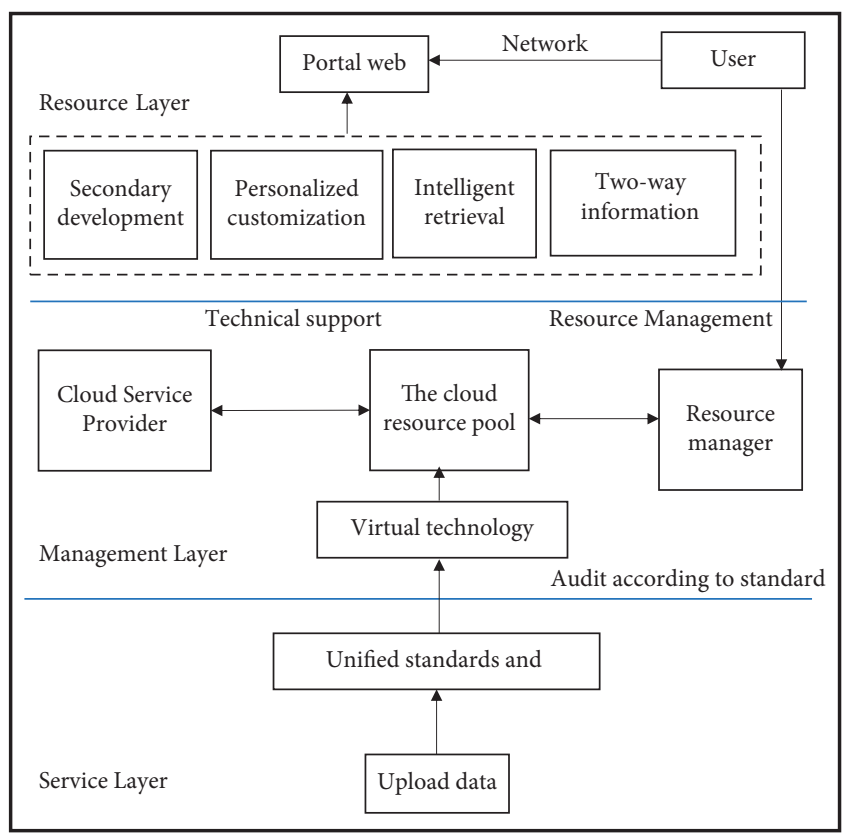

Figure 1: Platform architecture.

OpenStack toolsets, it can effectively manage a large number of resource pools (formed by computers, storage devices, and network resources). OpenStack is an open, free standard cloud computing platform. It is widely installed as infrastructure-as-a-service (IaaS) in both public and private clouds where resources and virtual servers are can be accessed by users [20]. The storage function architecture based on OpenStack is shown in Figure 2.

\section{Mobile Learning Community Resource Sharing}

In this study, the contact intensity between nodes was used to describe the similarity between nodes. Let $C_{(u, v)}$ represent the contact strength between node $u$ and node $v$, and then the contact strength can be computed as

$$
C_{(u, v)}=C_{(u, v) \text { old }}+\left(1-C_{(u, v) \text { old }}\right) \times C_{i},
$$

where $\mathrm{C}_{(u, v) \text { old }}$ represents the contact intensity between node $u$ and node $v$ in the last contact. $C_{i}$ is an initial value, which was uniformly set to 0.75 in this experiment. The recession component was computed using the following equation:

$$
C_{(u, v)}=C_{(u, v) \text { old }} \times \alpha^{t},
$$

where $\alpha$ is the decay index, which was set to 0.98 in this experiment.

The transmission component was computed as

$$
C_{(u, \omega)}=C_{(u, \omega) \text { old }}+\left(1-C_{(u, \omega) \text { old }}\right) \times C_{(u, 9)} \times C_{(\vartheta, \omega)} \times \beta .
$$

The parameter $\beta$ is used to describe the dependence between nodes, which was set to 0.25 in the experiment. 


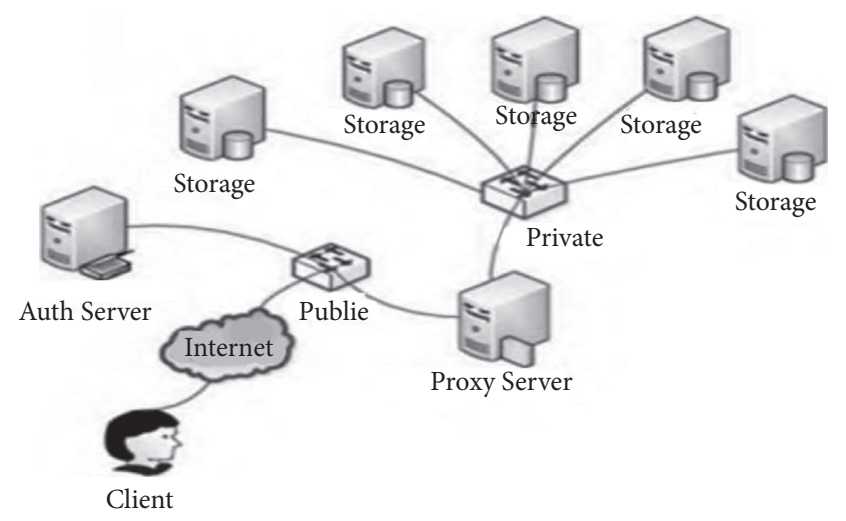

FIGURE 2: Storage architecture diagram.

The PageRank algorithm was used to calculate the centrality of nodes. Let $P_{(u)}$ represent the centrality of node $u$, and $P_{(u)}$ can be expressed as

$$
P_{(u)}=\frac{1-d}{n}+d \sum_{\vartheta \varepsilon N(u)} \frac{P_{(\vartheta)}}{N_{(\vartheta)}},
$$

where the parameter $d$ represents the decay factor, which was set to 0.3 in the current experiment, and $N_{(u)}$ represents the number of neighbors of node $u$.

According to the weighted square Euclidean distance measure between the sample and the cluster center, the objective function is minimized and the membership degree and cluster center are obtained. The clustering objective function was described as follows:

$$
\min L_{m}=\sum_{i=1}^{N} \sum_{j=1}^{K} z_{i j}^{m}\left\|x_{i}-V_{j}\right\|^{2} .
$$

Furthermore, the fuzzy membership and dissimilarity measure was employed to construct the objective function, find the minimum membership and cluster center of the objective function through iteration, and realize sample classification.

$$
\begin{aligned}
J & =\sum_{i=1}^{N} \sum_{j=1}^{K} u_{i j}^{m}\left[d^{2}\left(x_{i}, v_{j}\right)+G_{i j}\right], \\
G_{i j} & =\sum_{\beta=N_{I}} \frac{1}{d_{i \beta}+1}\left(1-u_{i \beta}\right)^{m} .
\end{aligned}
$$

Finally, the optimization solution expression of the objective function was computed as

$$
\begin{aligned}
u_{i j} & =\frac{1}{\sum_{l=1}^{K} d^{2}\left(x_{i}, v_{j}\right)+G_{i j} / d^{2}\left(x_{i}, v_{l}\right)+G_{i l}}, \\
v_{k} & =\frac{\sum_{j=1}^{K} u_{i j}^{m} x_{i}}{\sum_{j=1}^{K} u_{i j}^{m}} .
\end{aligned}
$$

3.1. Proposed Algorithm. Considering the self-organization characteristics in the person-to-person contact environment, a distributed node centrality algorithm is proposed in this study. When two nodes meet, they exchange their current centrality information and the number of neighbors (Algorithm 1: steps 5 and 6) and then update their centrality (Step 7) according to steps given in equation (5). The whole calculation process is described in Algorithm 1.

\section{Experimental Process and Performance Analysis}

4.1. Setting Up the Experimental Environment. In this study, a simulation platform based on VC.NET is developed. The whole platform integrates the common node movement model, resource query, sharing algorithm, and neighbor discovery. The overall interface of the system is mainly composed of the initial set of the system environment, the choice of node movement mode, and the algorithm of resource sharing. The settings of environment variables used in this system mainly include the realization of environment area, number of nodes, maximum communication distance of nodes, size of node buffer, neighborhood identification cycle, packet sending rate, and experiment duration. The simulation platform integrates three of the most representative movement models and a real dataset collected at the Korean Advanced Institute of Science and Technology (KAIST).

We employed a dataset provided by the Korean Academy of Sciences [11]. The dataset collected the movement tracks of 34 students over a period of nearly two years, generating a total of 92 files, each containing hundreds to thousands of independent system logs. This dataset contains information about the location of each student at different times. The specific format of data in each file is as follows: residence time, location $(x, y)$, where $x$ and $y$ represent the coordinate information of each student.

4.2. Experimental Performance Analysis. In this section, we analyzed the influence of user's sociality on the efficiency of the proposed educational resource sharing model. We investigated the influence of user similarity and centrality on the response time of the resource acquisition model. The resource acquisition response time refers to the time between a user making a request for a particular educational resource and the user receiving the resource. The resource response time of the system is represented by the average of the resource response times of all the users.

We first analyze the types and number of educational resources that are known to the users, that is, the users in the system know exactly what resources other users have. In this case, when a user initiates a resource sharing request message, the user receiving the message responds with a confirmation message if it has the resource available. If the resource is not available with the current user, the node forwards the request message to those nodes that are closely related to the resource owner (i.e., those nodes that are highly similar to the resource owner). When the other nodes receive the same request message, they operate similarly. To study the impact of node similarity on resource response time, we devise another strategy for comparative analysis: we 


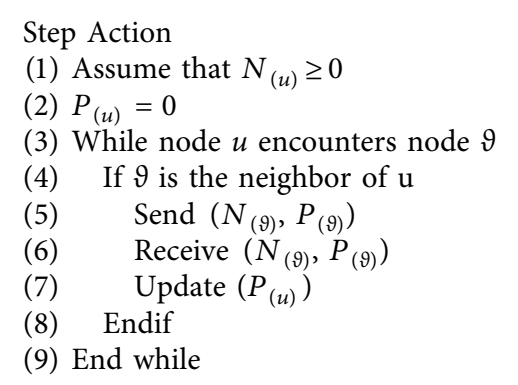

Algorithm 1: Node centrality.

randomly select $K$ nodes as relays. Finally, the response time of the resources in these two cases is calculated.

4.2.1. Average Response Time. Figure 3 shows the average response time when $K=8$. It is evident that the response time gradually gets longer with the progress of the experiment. The brown-colored line bars in the figure represent the time it takes for learners to obtain resources ( $k$ nodes are randomly selected as relays) after making resource requests in the learning group under normal circumstances. The blue bars indicate the response time of the resource when the request information is forwarded to members who are more similar to the resource owner. It can be seen that the time required to seek help from members with high similarity is $70 \%$ lower than the time required for normal communication (this is particularly obvious in $1200 \mathrm{~s}$ ).

4.2.2. Success Rate. Figure 4 shows the success rate of resource requests in both cases. It can be seen that the success rate of resource requests of nodes with high similarity is also higher than the success rate of resource acquisition by randomly selected nodes. For example, the success rate of high similarity response time is 0.8 as compared to the success rate of success rate with random $k$ point selection. Therefore, in the mobile learning community, when members know which members can help them or their friends are likely to help them, they can make requests directly to them, and it is a very convenient method to get resources.

The above analysis assumes that learners who have resources are known, and then they request these members to analyze their response time and success rate of obtaining resources. However, in most cases, learners may not know which nodes have resources, and in fact, there is no need to care about the owners of resources. Users care about access to the resource, not where it is stored. In this case, $K$ nodes with high centrality are selected to participate in resource request forwarding, that is, the resource requesting node sends the request information to the nodes with high centrality, and these nodes carry out the relay operation.

4.2.3. Effect of Node Selection. Figure 5 shows the success rate for different values of $k$. It can be seen that the success rates gradually decrease when the value of $k$ is increased

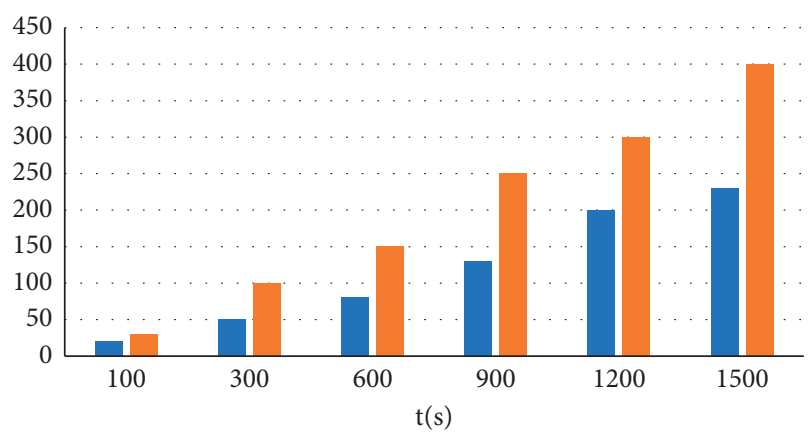

- Similarity Response Time

- K-Response Time

Figure 3: Average response time.

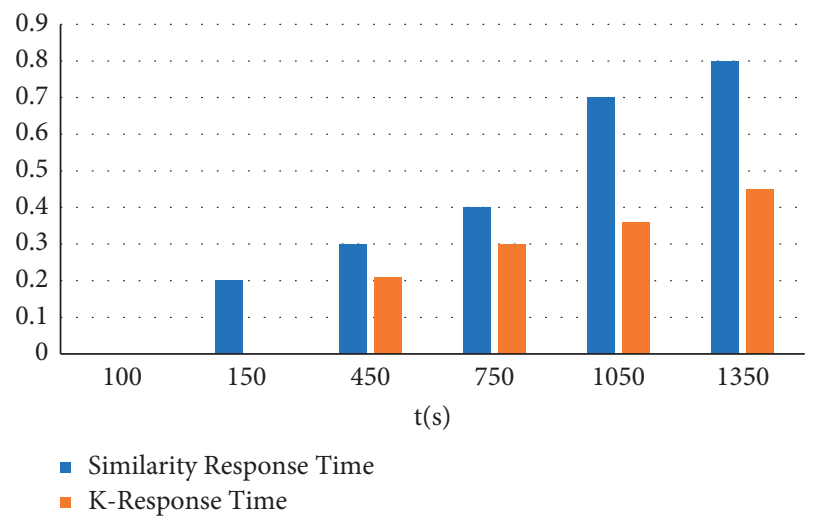

FIgURE 4: Comparison of similarity and success rate of randomly selected resource requests.

from 3 to 30 in the experiment. The results show that the method of $K$-node selection is adversely affected when the number of nodes for $K$-node selection increased as opposed to the proposed method. This validates that the method of high similarity is more superior to the method of $K$-node selection for educational resource sharing.

Based on the above experimental results, it can be concluded that in the learning community established in the WiFi environment, the shortest response time of interaction between members is $300 \mathrm{~s}$, and the average time is about $13 \mathrm{~min}$, which is acceptable. In addition, today's smartphones also provide a mobile push 


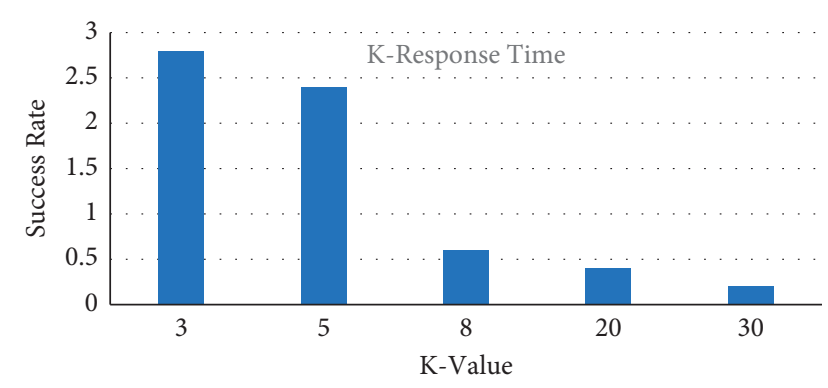

FIGURE 5: Effects of node selection.

notification function. For example, using WeChat, mobile QQ, and other software as the learning environment, the response time will be shorter, and community members can receive requests and reply in time. In this community, if learners ask questions to members with high centrality, the success rate of resource acquisition will be higher and the response time will be shorter. This is also related to the active degree of each member in the learning community. The higher the active degree is, the faster the corresponding help will be received, the shorter the response time is, and the longer the survival time of the community is.

\section{Conclusion}

In this study, an educational resource sharing algorithm based on WiFi is presented. Based on the analysis of the current teaching resource sharing system and its characteristics, an educational resource sharing system based on the service mode of the teaching sharing platform is designed. The system has a high success rate and low response time. The results show that in an environment where the resource owner is known, sending the resource request information to users with high similarity to the resource owner will reduce the response time of the resource and improve the success rate of the resource request. In an environment where the owner of the resource is unknown, sending the resource request information to those nodes will significantly reduce the efficiency of resource sharing. Encourage regional teaching units to provide broader collaboration and more comprehensive resource sharing.

\section{Data Availability}

The data used to support the findings of this study are available from the corresponding author upon request.

\section{Conflicts of Interest}

The authors declare that they have no conflicts of interest.

\section{References}

[1] Q. Chen, "Study on the architectural structures of the miniprogram based on Webpy+Apache HTTP server," Wireless Internet Technology, vol. 2017, no. 18, 51 pages, 2017.

[2] H. L. Fang, P. Ross, and D. Corne, "A promising genetic algorithm approach to job-shop scheduling, "Rescheduling, and Open-Shop Scheduling Problems," in Proceedings of the Fifth International Conference on Genetic Algorithms, pp. 375-382, San Mateo, CA, July 1993.

[3] J. R. Anderson, L. M. Reder, and H. A. Simon, "Situated learning and education," Educational Researcher, vol. 25, no. 4, pp. 5-11, 1996.

[4] C. Alvarez, M. Nussbaum, M. Recabarren, and F. Gomez Zaccarelli, "Teaching communication, interpersonal and decision-making skills in engineering courses supported by technology," International Journal of Engineering Education, vol. 25 , no. $4,2009$.

[5] S. Choi, H. Kim, H. Jang et al., "A framework for ensuring consistency of web services transactions," Information and Software Technology, vol. 50, no. 7-8, pp. 684-696, 2008.

[6] M. Younas, I. Awan, and D. Duce, "An efficient composition of web services with active network support," Expert Systems with Applications, vol. 31, no. 4, pp. 859-869, 2006.

[7] J. Didac, J. Anderson, and M. Milrad, "Enhancing mobile learning activities by the use of mobile virtual devices - some design and implementation issues," in Proceedings of the Intelligent Networking and Collaborative Systems, International Conference on, vol. 1, IEEE, Thessalonika, Greece, November 2010.

[8] Y. Hou, N. Wang, G. Mei, W. Xu, W. Shao, and Y. Liu, "Educational resource sharing platform based on blockchain network," in Proceedings of the 2019 Chinese Automation Congress (CAC), pp. 5491-5494, IEEE, Hangzhou, China, November 2019.

[9] L. Yao and X. Xiong, "Design a teaching resource sharing system in colleges based on cloud computing," in Proceedings of the Information Technology and Applications (ITA), pp. 374-377, IEEE, Chengdu, China, November 2013.

[10] N. Yalcin, Y. Altun, and U. Kose, "Educational material development model for teaching computer network and system management," Computer Applications in Engineering Education, vol. 23, no. 4, 2015.

[11] L. Nan and S. Li, "A review of mobile learning in China from 2006 to 2011," Vocational Education Forum, vol. 2012, no. 18, 57 pages, 2012.

[12] Z. Haofeng, L. Ruiping, and L. Ming, "Social network analysis of QQ virtual learning community," Modern Educational Technology, vol. 2009, no. 12, 83 pages, 2009.

[13] L. Geping, H. Zhixing, and Q. Yuhui, "Design and implementation of distance learning process evaluation system based on data mining," Online Education and Distance Education, vol. 2005, no. 7, 69 pages, 2005.

[14] G. Yun-nan, Z. Jinhua, and H. Xia-Lan, "The concept, measure and influence of social network: a literature review," Zhejiang Social Sciences, vol. 2015, no. 2, 132 pages, 2015.

[15] F. Hoadley and C. Ke, "Evaluating online learning communities," Educational Technology Research \& Development, vol. 57, no. 4, pp. 487-510, 2009.

[16] H. Zhixin and W. Yizhi, "Research on personalized learning system based on mobile learning," Education Informatization in China, vol. 2014, no. 11, 71 pages, 2014.

[17] A. Lindgren, A. Doria, and O. Schelén, "Probabilistic routing in intermittently connected networks," Service Assurance with Partial and Intermittent Resources, vol. 3126, pp. 239-254, 2004.

[18] L. Zhu, H. Li, and C. Hong, "Review on the research of knowledge sharing under open innovation model," Journal of Modern Information, vol. 38, no. 1, pp. 169-177, 2018.

[19] G. Shaoqing, H. Jianjun, and Y. Qingfei, "A review of mobile learning application in foreign countries," Research on Audiovisual Education, vol. 2011, no. 5, 109 pages, 2011. 
[20] R. Guha, "Flexible web service infrastructure for the development and deployment of predictive models," Journal of Chemical Information and Modeling, vol. 48, no. 2, pp. 456464, 2008. 